\title{
Corrigendum: Mechanism of Growth Regulation of Yeast Involving Hydrogen Sulfide From S-Propargyl-Cysteine Catalyzed by Cystathionine- $\gamma$-Lyase
}

\author{
Zhongkai Gu ${ }^{1 *}$, Yufan Sun ${ }^{2 \dagger}$, Feizhen $W^{1+}$ and Xiaomo $\mathrm{Wu}^{3 *}$ \\ ${ }^{1}$ The Institute of Biomedical Sciences, Fudan University, Shanghai, China, ${ }^{2}$ Key Laboratory of Medical Molecular Virology of \\ Ministries of Education and Health, Department of Medical Microbiology, School of Basic Medical Sciences, Fudan \\ University, Shanghai, China, ${ }^{3}$ Dermatology Institute of Fuzhou, Dermatology Hospital of Fuzhou, Fuzhou, China
}

Keywords: hydrogen sulfide, $\mathrm{H}_{2} \mathrm{~S}$ metabolism, fungal growth rate, SPRC, cystathionine- $\gamma$-lyase, fungal growth

\section{A Corrigendum on}

\section{OPEN ACCESS}

Approved by:

Frontiers Editorial Office,

Frontiers Media SA, Switzerland

*Correspondence:

Zhongkai Gu

zhongkaigu@fudan.edu.cn Xiaomo Wu

xiaomo.wu@gmail.com

tThese authors have contributed equally to this work

Specialty section:

This article was submitted to Antimicrobials, Resistance and

Chemotherapy,

a section of the journal

Frontiers in Microbiology

Received: 19 August 2021

Accepted: 26 August 2021

Published: 24 September 2021

Citation:

Gu Z, Sun Y, Wu F and Wu X (2021)

Corrigendum: Mechanism of Growth

Regulation of Yeast Involving

Hydrogen Sulfide From

S-Propargyl-Cysteine Catalyzed by

Cystathionine- $\gamma$-Lyase.

Front. Microbiol. 12:761240.

doi: 10.3389/fmicb.2021.761240
Mechanism of Growth Regulation of Yeast Involving Hydrogen Sulfide From $S$-Propargyl-Cysteine Catalyzed by Cystathionine- $\gamma$-Lyase

by Gu, Z., Sun, Y., Wu, F., and Wu, X. (2021). Front. Microbiol. 12:679563. doi: $10.3389 /$ fmicb.2021.679563

Xiaomo Wu was not included as an author in the published article. Her affiliation is "Dermatology Institute of Fuzhou, Dermatology Hospital of Fuzhou, Fuzhou, China". She is a corresponding author of the article. The corrected Author Contributions Statement appears below.

"ZG designed the total topic and all the experiments and took in charge of almost all the experiments and the data analyses. YS took in charge of the purification of Cys3p of budding yeast. FW took in charge of the direction on the data analyses. XW took in charge of the manuscript preparation. All the authors contributed to the article and approved the submitted version."

In the original article, we neglected to include the funder Key Clinical Specialty Discipline Construction Program of Fuzhou, 201807111, and Clinical Medicine Center Construction Program of Fuzhou, 2018080309 to Xiaomo Wu. The corrected Funding statement appears below.

"The study was funded in part by a grant from the National Key Basic Research Project of China [grant number: 2013CB531603]. This work was supported by Key Clinical Specialty Discipline Construction Program of Fuzhou (201807111) and Clinical Medicine Center Construction Program of Fuzhou (2018080309) to XW.”

The authors apologize for this error and state that this does not change the scientific conclusions of the article in any way. The original article has been updated.

Publisher's Note: All claims expressed in this article are solely those of the authors and do not necessarily represent those of their affiliated organizations, or those of the publisher, the editors and the reviewers. Any product that may be evaluated in this article, or claim that may be made by its manufacturer, is not guaranteed or endorsed by the publisher.

Copyright (c) $2021 \mathrm{Gu}$, Sun, Wu and Wu. This is an open-access article distributed under the terms of the Creative Commons Attribution License (CC BY). The use, distribution or reproduction in other forums is permitted, provided the original author(s) and the copyright owner(s) are credited and that the original publication in this journal is cited, in accordance with accepted academic practice. No use, distribution or reproduction is permitted which does not comply with these terms. 